\title{
TaTME, tips para iniciar la curva de aprendizaje
}

\author{
Adrián Mattacheo, ${ }^{1,2}$, Joaquín Tognelli'i, Julio Lococo ${ }^{3}$ \\ ${ }^{1}$ Hospital J. M. Ramos Mejía. CABA, Argentina. \\ ${ }^{2}$ Sanatorio Sagrado Corazón. CABA, Argentina. \\ ${ }^{3}$ Hospital Churruca-Visca. CABA, Argentina.
}

La cirugía para el cáncer de recto ha sido un procedimiento históricamente complejo. Múltiples factores explican las altas tasas reportadas de recurrencia local a través del tiempo. Además de factores biológicos inherentes a la enfermedad en sí, su ubicación en la pelvis, área anatómica rodeada de estructuras óseas, vasculares y nerviosas, así como de órganos genitourinarios en su relación anterior, condicionan esta situación. Al respecto, conocemos que las tasas de margen comprometido por el tumor durante la disección abdominal, se relacionan directamente con ese alto porcentaje de recaídas locales. Ya de por sí una situación desafiante para el cirujano, lo es mucho más en el caso de tumores voluminosos, especialmente en hombres con pelvis angostas y obesos.

La cirugía de TaTME (escisión total mesorrectal transanal) se difundió a partir 2010 con la publicación de Sylla, ${ }^{1}$ con la finalidad de subsanar las dificultades que fueron mencionadas, mediante el abordaje combinado ascendente-descendente.

Como contrapartida, considerando esta cirugía desde un nuevo y poco explorado abordaje, aparecieron nuevas y graves complicaciones relacionadas específicamente con el procedimiento. Al día de hoy, existe gran cantidad de reportes, cursos y conferencias relacionados con la técnica y con los resultados, ya sean oncológicos y/o, sobre todo, perioperatorios. Todos ellos han intentado plantear la seguridad de la cirugía en sus múltiples esferas.

En esta reseña, sin el objetivo de constituir un capítulo de técnica quirúrgica, intentaremos transmitir algunos conceptos aprendidos durante nuestra experiencia inicial en la cirugía de TaTME. Algunos de los cuales pueden resultar a simple vista evidentes, pero que no por eso deben escapar de nuestro control.

1. Tiempo: al planificar la cirugía, debemos asumir que estas son cirugías largas (aún contando con equipos simultáneos para los tiempos abdominal y transanal). Esto nos ayudará en la planificación de nuestra jornada quirúrgica, así como a dedicarle el tiempo necesario a los pasos más difíciles de la operación.

Los autores declaran ausencia de conflictos de interés.

Adrián Mattacheo

amattacheo@gmail.com

Recibido: mayo de 2020. Aceptado: junio de 2020.
2. Selección de los casos: creemos preferible iniciar la experiencia con lesiones de recto medio. Si bien esta técnica es ideal para lesiones en recto medio y bajo, las últimas acarrean el inconveniente adicional de la disección del cuerpo perineal en la cara anterior, donde se confunde el plano de disección con la capa muscular propia del recto y la próstata, adicionando tiempo quirúrgico por el temor a la lesión uretral o a la perforación del tumor.

3. Equipos simultáneos: emplear 2 equipos experimentados, uno realizando la disección abdominal y el otro, la transanal, trae evidentes beneficios en el tiempo operatorio. De ser esta la situación, la colocación de las piernas del paciente no requiere una posición de litotomía muy forzada. Una mínima elevación suficiente para colocar el dispositivo transanal, le brindará al equipo abdominal mayor libertad de movimientos, especialmente a la hora del descenso del ángulo esplénico. De igual manera, ambas torres pueden colocarse a la izquierda del paciente.

4. Dispositivo: hoy en día, existen 2 posibilidades a la hora de elegir el dispositivo transanal. Uno descartable (GelPOINT Path-Applied Medical, Rancho Santa Margarita, CA, USA), y el otro reutilizable (TEOKarl Storz, Tuttlingen, Alemania). Hemos utilizado ambos. Los dos tienen sus pros y sus contras. Últimamente hemos decantado la elección por el TEO por su confiabilidad y durabilidad. Aunque reservamos la utilización del GelPoint para los casos en los que la jareta es de más difícil realización.

5. Jareta: la confección de la jareta distal no puede ser subestimada. Para algunos autores, hacerlo incorrectamente, puede conducir a altas tasas de recurrencias tempranas en algunas series, opinión que compartimos. ${ }^{2}$ En primer lugar, si puede realizarse con un portaagujas largo de cirugía convencional a través del dispositivo transanal, se ahorrará tiempo. Es especialmente importante que los puntos se coloquen uno inmediatamente al lado del anterior, sin espacios entre ellos, evitando la espiralización de la sutura. Si la jareta no cierra herméticamente (esto se comprueba pasando una pinza de Maryland a través de la misma), debe ser rehecha las veces que sea necesario. 
6. Neumorrecto-neumopelvis: el efecto de bamboleo u oscilación presente en estas técnicas con insufladores convencionales puede mejorar mediante la interposición de una bolsa semirrígida (la colectora de orina), a expensas de una menor distensión de la cavidad. Por otra parte, existen reportes de embolias gaseosas en pacientes sometidos a esta cirugía. ${ }^{3}$ La primera manifestación de esta complicación es la amputación de la curva de capnografía, situación que debe ser monitorizada por el anestesiólogo y transmitirse al cirujano, a fin de tomar las conductas correspondientes. Hubo en nuestra experiencia un caso que, por colapso hemodinámico, requirió la suspensión del neumorrecto y la conversión inmediata de la cirugía.

7. Espesor de la pared: es importante familiarizarse con la capa muscular propia, y su espesor. La confusión puede conducir a la disección de la pared rectal, comprometiendo de esta manera uno de los objetivos primordiales de esta técnica: mejorar la calidad de la pieza quirúrgica. Identificar la capa muscular y seccionarla en toda su circunferencia desde el inicio permitirá encontrar el plano de disección hacia la fascia mesorrectal con más facilidad. Encontramos más sencilla la disección posterior y anterior indistintamente, antes que la lateral, en la medida que se vaya presentando de acuerdo con cada caso. En la mujer, el tacto vaginal puede ayudar a la identificación del plano correcto de disección.
8. Extracción: luego de completada la disección, siempre extraemos la pieza por el abdomen. Generalmente a través de una pequeña incisión de Pfannenstiel. Evitamos la extracción a través del ano para respetar la indemnidad del mesorrecto y la sobredistensión del esfínter.

Por último, pero no por eso menos importante, consideramos fundamental remarcar algunos conceptos que pudimos experimentar, relacionados con la necesidad de preparación y de volumen de casos. Son tal vez los más importantes de ellos. Haber realizado cursos teóricoprácticos, así como ser asistidos por un cirujano experimentado en los primeros casos, fue fundamental a la hora de subsanar los problemas e inquietudes que, necesariamente, surgen al encarar una cirugía a la que no estamos habituados. Puntualmente, es frecuente precisar de una guía a la hora de reconocer los planos durante la disección transanal. Esto se ve reflejado en los tiempos operatorios, pero también en la disminución en la ocurrencia de complicaciones. Por otra parte, si bien no existe un consenso sobre el número de procedimientos requeridos para superar la curva de aprendizaje, se la ha contemplado entre 20 y 50 casos. ${ }^{4}$ Por lo cual, no tener un bagaje de volumen de cirugía de cáncer de recto, ni de cirugía transanal suficiente, tendrá necesariamente su efecto retardador en la curva.

\section{REFERENCIAS}

1. Sylla P, Rattner DW, Delgado S, Lacy AM. NOTES transanal rectal cancer resection using transanal endoscopic microsurgery and laparoscopic assistance. Surg Endosc 2010;24:1205-10.

2. Francis N, Penna M, Mackenzie H, Carter F, Hompes R. Consensus on structured training curriculum for transanal total mesorectal excision (TaTME). Surg Endosc 2017;31:2711-19.

3. Dickson EA, Penna M, Cunningham C, Ratcliffe FM, Chantler
J, Crabtree NA, et al. Carbon dioxide embolism associated with transanal total mesorectal excision surgery: A report from the international registries. Dis Colon Rectum. 2019 Jul;62(7):794-801.

4. Adamina M, Buchs NC, Penna M, Hompes R. St. Gallen consensus on safe implementation of transanal total mesorectal excision. Surg Endosc 2018;32:1091-103. 